\title{
Current Concepts of Inguinal Hernia Repair
}

\author{
Ferdinand Köckerling ${ }^{a} \quad$ Maarten P. Simons ${ }^{b}$ \\ aDepartment of Surgery and Center for Minimally Invasive Surgery, Academic Teaching Hospital of Charité Medical School, \\ Vivantes Hospital, Berlin, Germany; \\ ${ }^{b}$ Department of Surgery, Onze Lieve Vrouwe Hospital, Amsterdam, the Netherlands
}

\section{Keywords}

Inguinal hernia - Totally extraperitoneal patch plasty . TEP . Transabdominal preperitoneal patch plasty .

TAPP · Lichtenstein · Tailored approach

\section{Summary}

With more than 20 million patients annually, inguinal hernia repair is one of the most often performed surgical procedures worldwide. The lifetime risk to develop an inguinal hernia is $27-43 \%$ for men and $3-6 \%$ for women. In spite of all advances, $11 \%$ of all patients suffer from a recurrence and $10-12 \%$ from chronic pain following primary inguinal hernia repair. By developing evidencebased guidelines and recommendations, the international hernia societies aim to improve the outcome of inguinal hernia repair due to standardization of care. From a total of more than 100 different repair techniques for inguinal and femoral hernias, classified as tissue repair, open mesh repair, and laparo-endoscopic mesh repair, the new International Guidelines of the HerniaSurge Group only recommend the totally extraperitoneal patch plasty (TEP), transabdominal preperitoneal patch plasty (TAPP), and Lichtenstein techniques. Since a generally accepted technique suitable for all inguinal hernias does not exist, surgeons should provide both an anterior open (Lichtenstein) and a posterior laparo-endoscopic (TEP or TAPP) approach option. The guidelines strongly recommend that surgeons tailor the treatment of inguinal hernias based on expertise, local/national resources, and patient- and hernia-related factors. A tailored approach in inguinal hernia repair should pay heed to the patient- and hernia-related factors, unilateral hernia in men and women, bilateral hernia, recurrent hernia, scrotal hernia, previous pelvic and lower abdominal surgery, severe cardiac or pulmonary comorbidities, and incarcerated hernia.

(C) 2018 S. Karger GmbH, Freiburg

\section{Introduction}

Worldwide, inguinal hernia repair is one of the most common surgeries, being performed in more than 20 million people annually $[1,2]$. The lifetime occurrence of groin hernia - viscera or adipose tissue protrusions through the inguinal or femoral canal - is $27-43 \%$ in men and $3-6 \%$ in women $[1,2]$. Inguinal hernias are almost always symptomatic, and the only cure is surgery [1]. A minority of patients is asymptomatic; however, even a watch-andwait approach in this group results in surgery in approximately $70 \%$ within 5 years $[1,3]$. Surgical treatment is successful in the majority of cases [1]. The expected rate of recurrence following inguinal hernia repair is still $11 \%$ today [4]. Only $57 \%$ of all inguinal hernia recurrences occurred within 10 years after the previous hernia operation. Some of the remaining $43 \%$ of all recurrences happened only much later, even after more than 50 years [4]. A further problem after inguinal hernia repair is chronic pain lasting more than 3 months, occurring in $10-12 \%$ of all patients [1]. Approximately $1-3 \%$ of patients have severe chronic pain with long-term disability, thus requiring treatment [1].

With the aim to improve the outcome of inguinal hernia repair by means of standardizing care, the European Hernia Society (EHS), the International Endohernia Society (IEHS), and the European Association for Endoscopic Surgery (EAES) published guidelines [5-9]. These three societies began collaborating in 2014 and developed a universal set of guidelines for groin hernia management [1], called International Guidelines for Groin Hernia Management by the HerniaSurge Group, with representatives from all continental hernia societies (EHS, IEHS, EAES, Americas Hernia Society (AHS), Asia Pacific Hernia Society (APHS), Afro Middle East Hernia Society (AMEHS), and Australasian Hernia Society). This article summarizes parts of the International Guidelines and where indicated offers results from other prior guidelines. It also includes recent literature published after the deadline date of July 1 , 2015 used for the International Guidelines.

\section{KARGER}

(๑) 2018 S. Karger GmbH, Freiburg

Fax +497614520714
Prof. Dr. med. Ferdinand Köckerling 


\section{Evidence-Based Selection of Techniques}

Since the epoch-making contribution of Bassini in 1888, more than 100 different techniques for repair of inguinal and femoral hernias have been described [10], mainly characterized as tissue, open mesh, and laparo-endoscopic mesh repair techniques.

In their International Guidelines the HerniaSurge Group strongly recommends a mesh-based repair technique for patients with inguinal and/or femoral hernia [1].

In a Cochrane review comparing the open non-mesh Shouldice technique with other open techniques, a total of 2,566 hernias from 16 trials were analyzed in the open non-mesh Shouldice group and compared with 1,121 open mesh and 1,608 open non-mesh techniques [11]. The recurrence rate with Shouldice techniques was higher than with open mesh techniques (odds ratio (OR) 3.80; 95\% confidence interval (CI) 1.99-7.26) but lower than with other non-mesh techniques (OR 0.62; 95\% CI 0.45-0.85) [11]. There were no significant differences in chronic pain, complications, and postoperative stay [11].

On the basis of this meta-analysis, the HerniaSurge Group recommends the Shouldice technique in non-mesh inguinal hernia repair [1]. Non-mesh repair for inguinal hernia can be suggested in cases where the patient refuses a mesh, after shared decision making with the patient, or in low-resource settings with non-availability of meshes [1].

In two meta-analyses comparing the open mesh 'plug-and-patch' and Prolene Hernia System (PHS) techniques with the open Lichtenstein technique, no difference was found in terms of time to return to work, complications, chronic pain, and hernia recurrence $[12,13]$. Although the HerniaSurge Group also states that the recurrence rate and postoperative chronic pain rate are comparable between plugand-patch/PHS and the Lichtenstein technique, they do strongly not recommend plug-and-patch/PHS because of the excessive use of foreign material, the need to enter both the anterior and posterior planes, and the additional cost [1]. To replace the standard flat mesh in the Lichtenstein technique is strongly not recommended [1]. Furthermore, in open inguinal hernia repair, there is insufficient evidence to favor preperitoneal mesh repair over Lichtenstein repair [1]

For comparison of the best open technique with the laparo-endoscopic techniques transabdominal preperitoneal patch plasty (TAPP) and totally extraperitoneal patch plasty (TEP), five metaanalyses are available [14-18]. Only in one meta-analysis, TEP and TAPP were jointly compared with the best open mesh technique Lichtenstein [17]. No difference in the recurrence rates was found; however, a lower incidence of wound infection, an earlier return to normal activities or work, and a lesser incidence of chronic pain syndrome could be detected [17]. Two meta-analyses comparing only TEP and one comparing only TAPP with the Lichtenstein technique could not deliver sufficient evidence to determine the greater effectiveness of one over the other technique [19-21].

In a prospectively documented registry-based study comparing 10,555 Lichtenstein repairs with 6,833 TEP repairs in primary unilateral inguinal hernias in men [22], no difference in recurrence rate, complication-related reoperation rate, and chronic pain rate requiring treatment was detected in a multivariable analysis. How- ever, TEP was found to have benefits in regard to postoperative complication rate $(\mathrm{p}<0.001)$, pain at rest rate $(\mathrm{p}=0.011)$, and pain on exertion rate $(\mathrm{p}<0.001)$ [22].

When comparing TEP and TAPP with the Lichtenstein technique, a registry-based, propensity score-matched comparison of 57,906 patients with primary unilateral inguinal hernia repair revealed significantly less postoperative complications, complication-related reoperations, pain at rest, and pain on exertion in favor of TEP and TAPP [23]. TEP showed disadvantages in terms of intraoperative complications [23].

On the basis of the existing evidence, the HerniaSurge Group recommends a laparo-endoscopic technique for male patients with primary unilateral inguinal hernia because of lower postoperative pain incidence and comparable complication-related reoperations, provided that a surgeon with specific and sufficient expertise is available. However, there are patient and hernia characteristics that warrant the Lichtenstein technique as first choice [1]

Meta-analyses and registry studies comparing the laparo-endoscopic techniques TEP and TAPP demonstrate comparable outcomes [23-27]. Therefore, the HerniaSurge Group recommends in their International Guidelines that the choice of the techniques TEP or TAPP should be based on the surgeon's skills, education, and experience [1].

In conclusion, the new International Guidelines of the HerniaSurge Group recommend only the open mesh technique Lichtenstein and the laparo-endoscopic mesh techniques TEP and TAPP as repair techniques in inguinal hernia surgery. The non-mesh open technique Shouldice is only indicated when a patient refuses a mesh and/or after a shared decision making with a patient or when a mesh is not available [1]. The International Guidelines recommend that more studies be performed researching the value of tissue repair in certain patient categories like small lateral hernias in young patients [1]. Also more evidence is needed to support management in women and certain specific types like occult and potential bilateral inguinal hernia [1].

\section{Evidence-Based Tailored Approach}

In the International Guidelines of the HerniaSurge Group it is strongly recommended that surgeons tailor the treatment of inguinal hernias based on expertise, local/national resources, and patientand hernia-related factors [1]. Since a generally accepted technique suitable for all inguinal hernias does not exist, surgeons should provide both an anterior and a posterior approach option [1].

A tailored approach in inguinal hernia repair should distinguish between the following clinical situations: primary unilateral inguinal hernia in men and in women, primary bilateral inguinal hernia in men and in women, primary scrotal inguinal hernia, primary inguinal hernia after previous pelvic and lower abdominal surgery (radical prostatectomy, cystectomy, vascular surgery, low anterior resection of the rectum, previous gynecological operations, and ascites), severe cardiac or pulmonary risk factors requiring local or spinal anesthesia, recurrent inguinal hernia, and emergency surgery for incarcerated or strangulated inguinal hernia [28]. 


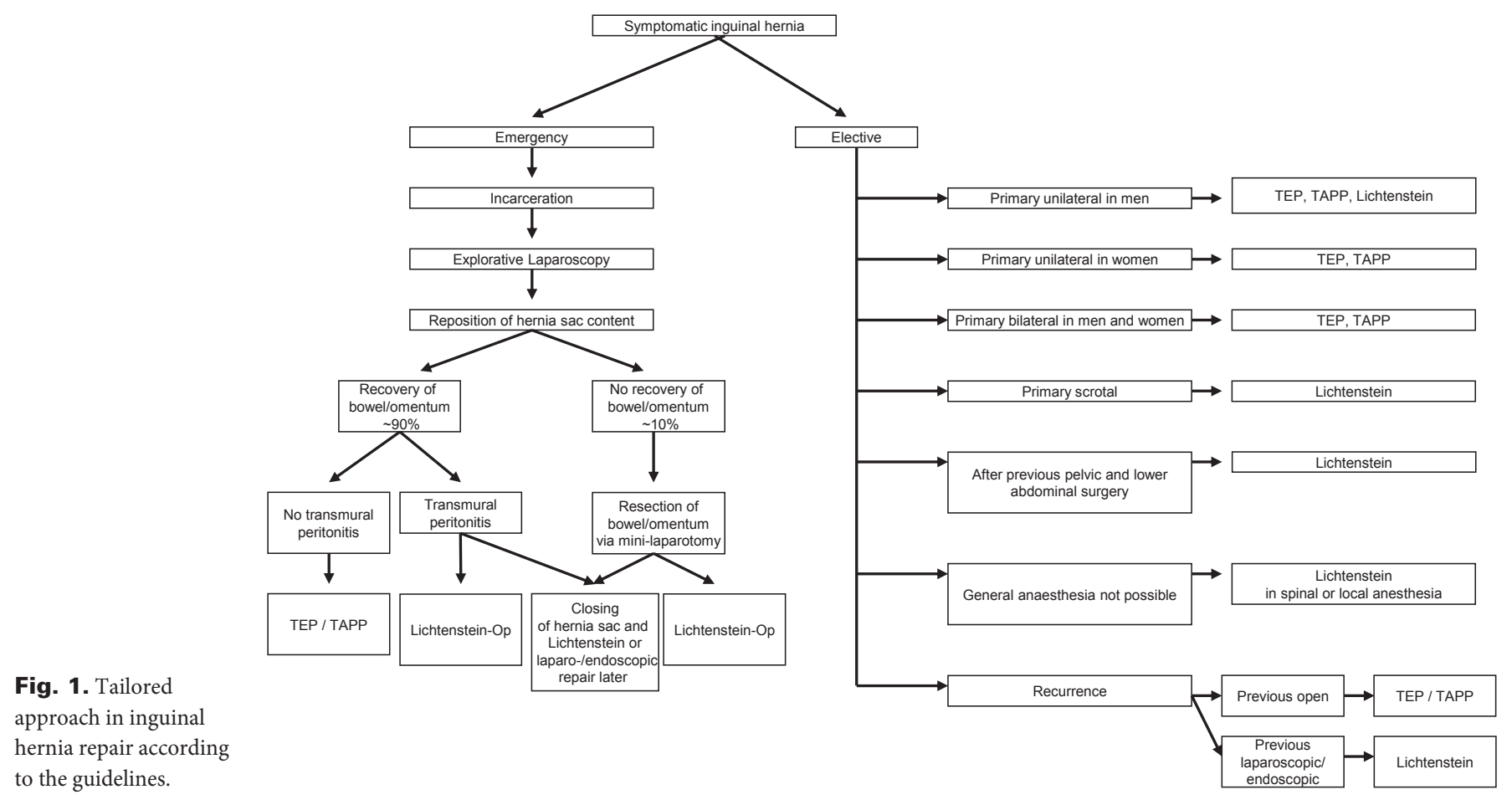

\section{Primary Unilateral Inguinal Hernia in Men}

On the basis of the existing evidence [5-27] for male patients with primary unilateral inguinal hernia repair (fig. 1), TEP or TAPP technique is recommended because of a lower risk of chronic pain and less postoperative complications, provided a surgeon with specific and sufficient expertise is available [1]. The guidelines recommended the best open mesh technique Lichtenstein as an alternative [1, 5-9].

\section{Primary Unilateral Inguinal Hernia in Women}

No systematic review or randomized controlled trials specifically address groin hernia repair in women (fig. 1) [1]. In a systematic review and meta-analysis of observational studies concerning patientrelated risk factors for recurrence, female sex was found to be a significant risk factor for recurrence after inguinal hernia surgery [29]. A study from the Danish Hernia Database demonstrated a 15-fold greater incidence of femoral hernias after inguinal hernia repair compared with spontaneous incidence [30]. These femoral recurrences occurred earlier than inguinal recurrences, suggesting that they were possibly femoral hernias overlooked at the primary operation [30]. All femoral recurrences occurred after a previous open anterior operation [31]. Laparoscopic repair of a femoral hernia reduces the risk of reoperation for a recurrence compared with open repair [32]. In women with a groin hernia, a femoral hernia should always be excluded by laparoscopy or by open exploration of the preperitoneal space [33].

Therefore, all guidelines $[1,5-9]$ strongly recommend a laparoendoscopic repair (TEP, TAPP) in women with groin hernias, provided that expertise is available. The only alternative is the careful exclusion of a femoral hernia by opening the transversalis fascia during an open anterior procedure and conversion to a preperitoneal mesh technique if a femoral hernia is found (fig. 1).

\section{Primary Bilateral Inguinal Hernia in Men and Women}

Two prospective randomized trials compared laparoscopic versus open mesh repair in bilateral inguinal hernia (fig. 1) [34, 35]. Laparoscopic bilateral inguinal hernia repair was significantly quicker as well as less painful and allowed an earlier return to work. Other comparative studies found additional advantages in terms of postoperative complications and hospital stay in favor of the laparoscopic approach [36-38]. Although high-level evidence is missing, it seems self-evident since the advantages of laparo-endoscopic repair (faster recovery, lower risk of chronic pain, and cost-effectiveness) increase when performing two hernia repairs via the same three keyhole incisions [1]. In all guidelines a strong recommendation is given that primary bilateral inguinal hernias should be repaired laparo-endoscopically, provided surgeons with specific expertise and sufficient resources are available [1, 5-9].

In a registry-based comparison of 6,700 unilateral and 2,695 bilateral inguinal hernia repairs in the TEP technique, a significantly higher intraoperative bladder injury as well as complication-related reoperation rate constitute a difference in the perioperative outcome between unilateral and bilateral TEP [39]. Comparing 10,887 TAPP procedures for unilateral and 4,289 for bilateral inguinal hernia repair, multivariable analysis confirmed a highly significant difference to the disadvantage of bilateral TAPP due to an increased complication-related reoperation rate [40]. Based on these results, prophylactic operation of the healthy other groin should not be performed [39].

\section{Primary Scrotal Hernia}

In the guidelines of the EAES, scrotal hernias are classified as being a complex condition [9]. For scrotal hernia, only highly experienced laparo-enoscopic hernia surgeons should opt for a mini- 
mally invasive technique $[7-9,28]$. The challenge in scrotal hernia repair is to ensure complete dissection of the large hernia sac from the inguinal canal and scrotum $[7,8,28]$. Failure to remove a large part of the hernia sac will generally result in persistent seroma formation [7, 8, 28]. Endoscopic control of bleeding during scrotal hernia repair often is also very difficult when dissecting the hernia sac from the spermatic cord structures $[7,8,28]$. Therefore, there is a higher incidence of postoperative secondary hemorrhage and hematoma [7, 8, 28]. Accordingly, the EHS guidelines recommend the open mesh technique as the procedure of choice for scrotal hernia $[5,6,28]$. HerniaSurge Group recommends individualization (open or TAPP) in large scrotal and/or irreducible hernia [1].

Primary Inguinal Hernia after Previous Pelvic Operations (Radical Prostatectomy, Cystectomy, Gynecologic Operation, Vascular Operations, Low Anterior Resections, and Ascites)

In these very complex situations (fig. 1), the guidelines of the IEHS and the EAES also recommend that only very experienced laparo-endoscopic hernia surgeons should opt for a minimally invasive procedure $[7-9,28]$. Following major lower abdominal and pelvic surgery, HerniaSurge and the EHS therefore recommend the open-mesh technique Lichtenstein $[1,5,6,28]$. The open mesh approach also presents the least risk in the presence of liver cirrhosis with ascites or for patients on peritoneal dialysis [28].

Primary Inguinal Hernia Repair in Patients with Severe Cardiac or Pulmonary Risk Factors

Based on the recommendations of HerniaSurge and the EHS, the open mesh technique Lichtenstein under local anesthesia is the preferred technique when general anesthesia is not possible for patients with an American Society of Anesthesiologists (ASA) score III or IV because of cardiac or pulmonary risk factors (fig. 1) [1, 5, $6,28]$.

HerniaSurge states that when compared with general anesthesia, local anesthesia is associated with faster mobilization, earlier hospital discharge, lower hospital and total healthcare costs, and fewer complications such as urinary retention and early postoperative pain [1]. However, when surgeons inexperienced in its use are to administer local anesthesia, more hernia recurrences might result $[1,41]$. When compared with regional anesthesia, local anesthesia is associated with earlier hospital discharge, lower hospital and total healthcare costs, and a lower incidence of urinary retention [1].

In a more recent prospective randomized trial comparing TEP under general anesthesia with Lichtenstein using local anesthesia in 384 patients, patients operated with TEP experienced less long-term postoperative pain and less limitation in their ability to exercise [42].

In another prospective randomized trial with 72 patients, Lichtenstein repair under local anesthesia was as good as TEP under general anesthesia [43].

In a study randomly dividing 60 male patients with unilateral inguinal hernia into a group of Lichtenstein repair under local anesthesia and a group with spinal anesthesia, the time spent in local anesthesia was higher [44]. Intraoperative pain was higher in local anesthesia than with spinal anesthesia. There was no difference in postoperative pain. Postoperative complications occurred more often in the spinal anesthesia group [44].

A comparable study randomly divided 50 patients with Lichtenstein repair to local or spinal anesthesia [45]. The authors concluded that tension-free mesh repair of inguinal hernias under local anesthesia is simple, safe, and cost-effective, with very low rates of complications and a speedy discharge [45].

In patients with a symptomatic inguinal hernia and higher risk of general anesthesia due to cardiac or pulmonary comorbidities, Lichtenstein repair in local or spinal anesthesia is recommended. Local anesthesia seems to come along with less complications than spinal anesthesia. Additionally, many patients with cardiac complications regularly take thrombocyte aggregation inhibitors, thus increasing the risk of bleeding and complications from spinal anesthesia. Therefore, the decision between local and spinal anesthesia should be made based on patient-related factors.

\section{Recurrent Inguinal Hernia}

The rate of recurrent inguinal hernias (fig. 1) is still 11\% today [4]. In the largest [46] of five meta-analyses [46-50] comparing laparo-endoscopic versus open repair of recurrent hernias following previous open non-mesh and mesh procedures, the minimally invasive technique was associated with less wound complications and a faster recovery to normal activity, whereas the re-recurrence rate was comparable between these two methods. In a registrybased study comparing TEP and TAPP for recurrent inguinal hernia repair, the results concerning intraoperative complications, complication-related reoperations, re-recurrences, pain at rest, pain on exertion, or chronic pain requiring treatment were equivalent [51]. Accordingly, all guidelines [1,5-9] recommend recurrent inguinal hernia repair following previous open surgery in the TEP or TAPP technique, since the operation is performed in an anatomic layer between the peritoneum and the abdominal wall in which no previous dissection has been performed [1, 5-9, 28].

A laparo-endoscopic approach for recurrence following a previous TEP or TAPP calls for widespread experience of minimally invasive inguinal hernia surgery and is also classified as constituting a complex situation $[1,5-9,28]$. It is recommended that a Lichtenstein procedure should be performed in such a situation [1, 5-9, 28].

In a registry study, $90.5 \%$ of patients out of 2,482 laparo-endoscopic recurrent repair operations and only $38.5 \%$ of patients out of the 2,330 open recurrent repair procedures were operated on in accordance with the guidelines [52]. The authors concluded that for recurrent inguinal hernia repair, the guidelines are not yet being observed to the extent required [52]. Paying heed to the guidelines, comparison of perioperative and 1-year outcome for laparo-endoscopic and open repair for primary versus recurrent male unilateral inguinal hernia showed significant differences to the disadvantage of the recurrent operation $[53,54]$.

\section{Emergency Surgery for Incarcerated Inguinal Hernias}

In a systematic review of 7 studies with 328 patients treated exclusively by means of a laparoscopic approach, the results were acceptable [55]. The average operating time was $61.3 \pm 12.3 \mathrm{~min}$, the 
number of conversions 6 , and the average hospital stay $3.8 \pm 1.2$ days. Furthermore, 25 minor and 9 major complications occurred, and 17 bowel resections were performed either laparoscopically or through a mini-laparotomy [55].

In the guidelines of the World Society of Emergency Surgery (WSES) it is therefore recommended that repair of incarcerated hernias (fig. 1) may be performed with a laparoscopic approach [56].

The choice of technique for repair is based on the contamination of the surgical field, the size of the hernia, and the experience of the surgeon [56]. Prosthetic repair with synthetic mesh is recommended for patients with intestinal incarceration and no signs of intestinal strangulation or concurrent bowel resection (clean surgical field) [56].

If diagnostic laparoscopy revealed a strangulation, the bowel or greater omentum can be withdrawn from the hernia sac, making an incision into the cranial hernia ring, if necessary [28]. Next, a decision must be taken as to whether parts of the omentum and/or intestines should be resected [28]. In approximately $90 \%$ of cases, the data show that this is not necessary as the organs recover after repositioning into the abdominal cavity [28]. Then, inguinal hernia repair can be carried out when no transmural surgical field contamination is present. Depending on the expertise of the surgeon, this can be done as a laparo-endoscopic procedure (TEP or TAPP) or in the Lichtenstein technique [28]. The guidelines of the HerniaSurge Group recommend similarly on clean elective hernia surgery mesh repair in emergent clean inguinal hernia repair [1].

If the surgical field is potentially contaminated, there are different options for treatment. The hernia sac can first be closed with a suture and the hernia repair in open or laparo-endoscopic technique can be postponed to a later time [28]. Alternatively, the inguinal hernia can be repaired simultaneously in a different anatomic layer as open mesh repair in the Lichtenstein technique. If intestinal resection is required, simultaneous repair of the inguinal hernia should be avoided, opting instead for repair at a later stage [28].

In the guidelines of the WSES for emergency hernia repair in potentially contaminated surgical fields due to strangulation and/ or concurrent bowel resection, a suture repair is recommended when the hernia defect in question is small. Synthetic mesh repair may be performed, but with caution. Biological meshes may be a valid option but merit a detailed cost-benefit analysis [56].

\section{Conclusions}

- Patients with primary inguinal hernia repair still develop recurrence in $11 \%$ and chronic pain in $10-12 \%$ today.
- To improve the outcome of inguinal hernia repair, the international hernia societies have published evidence-based guidelines for the standardization of care.

- From a total of more than 100 different techniques for the repair of inguinal and femoral hernias, the new International Guidelines of the HerniaSurge Group, with representatives from all continental hernia societies, only recommend the open mesh technique Lichtenstein and the laparo-endoscoic mesh techniques TEP and TAPP.

- Since a generally accepted technique suitable for all inguinal hernias does not exist, surgeons should provide both an anterior (Lichtenstein) and a posterior (TEP or TAPP) approach option for tailoring of the treatment.

- In primary unilateral inguinal hernia in men and women as well as in bilateral cases, the laparo-endoscopic approach (TEP, TAPP) is the first choice, provided the surgeon has sufficient expertise.

- A recurrent inguinal hernia after previous open repair should be repaired in a laparo-endoscopic approach and after previous laparo-endoscopic repair in the Lichtenstein technique.

- In large scrotal hernias, the Lichtenstein technique offers advantages.

- After previous pelvic or lower abdominal operation, the open mesh technique Lichtenstein is favored in terms of risk reduction.

- In patients with severe cardiac and/or pulmonary comorbidities with high risk for general anesthesia, a Lichtenstein approach under local or spinal anesthesia has advantages.

- In emergency inguinal hernia repair without contamination of the operative field, a laparo-endoscopic or Lichtenstein repair can be performed after laparoscopic repositioning of the incarcerated organs.

- When in emergency inguinal repair bowel resection is necessary or a contamination present, different approaches depending on the individual situation are possible: closing only the hernia sac and repair of the hernia later, tissue repair in Shouldice technique, and Lichtenstein technique with biologic or biosynthetic mesh.

\section{Disclosure Statement}

FK is a member of the HerniaSurge Group and the EHS. The author has no other conflict of interest. MPS is a member of the HerniaSurge group and the EHS and has no other conflict of interest.

\section{References}

1 HerniaSurge Group: International guidelines for groin hernia management. Hernia 2018;22:1-165.

2 Kingsnorth A, LeBlanc K: Hernias: inguinal and incisional. Lancet 2003;362:1561-1571.
3 Fitzgibbons RJ, Ramanan B, Arya S, Tumer SA, Li X, Gibbs JO, Reda DJ; Investigators of the Original Trial: Long-term results of a randomized controlled trial of a nonoperative strategy (watchful waiting) for men with minimally symptomatic inguinal hernias. Ann Surg 2013;258:508-515.
4 Köckerling F, Koch A, Lorenz R, Schug-Pass C, Stechemesser B, Reinpold W: How long do we need to follow-up our hernia patients to find the real recurrence rate? Front Surg 2015;2:24. 
5 Simons MP, Aufenacker T, Bay-Nielsen M, Bouillot JL, Campanelli G, Conze J, de Lange D, Fortelny R, Heikkinen T, Kingsnorth A, Kukleta J, Morales-Conde S, Nordin P, Schumpelick V, Smedberg S, Smietanski M, Weber G, Miserez M: European Hernia Society guidelines on the treatment of inguinal hernia in adult patients. Hernia 2009;13:343-403.

6 Miserez M, Peeters E, Aufenacker T, Bouillot JL, Campanelli G, Conze J, Fortelny R, Heikkinen T, Jorgensen LN, Kukleta J, Morales-Conde S, Nordin P, Schumpelick V, Smedberg S, Smietanski M, Weber G, Simons MP: Update with level 1 studies of the European Hernia Society guidelines on the treatment of inguinal hernia in adult patients. Hernia 2014;18:151-163.

7 Bittner R, Arregui ME, Bisgaard T, et al: Guidelines for laparoscopic (TAPP) and endoscopic (TEP) treatment of inguinal Hernia (International Endohernia Society (IEHS)). Surg Endosc 2011;25:2773-2843.

8 Bittner R, Montgomery MA, Arregui E, et al: Update of guidelines on laparoscopic (TAPP) and endoscopic (TEP) treatment of inguinal hernia (International Endohernia Society). Surg Endosc 2015;29:289-321.

9 Poelman MM, van den Heuvel B, Deelder JD, et al: EAES Consensus Development Conference on endoscopic repair of groin hernias. Surg Endosc 2013;27: 3505-3519.

10 Bendavid R: New techniques in hernia repair. World J Surgery 1989;13:522-531.

11 Amato B, Moja L, Panico S, Persico G, Rispoli C, Rocco N, Moschetti I: Shouldice technique versus other open techniques for inguinal hernia repair. Cochrane Database Syst Rev 2012;(4):CD001543.

12 Zhao G, Gao P, Ma B, Tian J, Yang K: Open mesh techniques for inguinal hernia repair: a meta-analysis of randomized controlled trials. Ann Surg 2009;250: $35-42$.

13 Li J, Ji Z, Li Y: Comparison of mesh-plug and Lichtenstein for inguinal hernia repair: a meta-analysis of randomized controlled trials. Hernia 2012;16:541-548.

14 Chung RS, Rowland DY: Meta-analyses of randomized controlled trials of laparoscopic vs conventional inguinal hernia repairs. Surg Endosc 1999;13:689-694.

15 EU Hernia Trialists Collaboration: Laparoscopic compared with open methods of groin hernia repair: systematic review of randomized controlled trials. $\mathrm{Br} \mathrm{J}$ Surg 2000;87:860-867.

16 Memon MA, Cooper NJ, Memon B, Memon MI, Abrams KR: Meta-analysis of randomized clinical trials comparing open and laparoscopic inguinal hernia repair. Br J Surg 2003;90:1479-1492.

17 Schmedt CG, Sauerland S, Bittner R: Comparison of endoscopic procedures vs Lichtenstein and other open mesh techniques for inguinal hernia repair: a metaanalysis of randomized controlled trials. Surg Endosc 2005;19:188-189.

18 O'Reilly EA, Burke JP, O'Connell PR: A meta-analysis of surgical morbidity and recurrence after laparoscopic and open repair of primary unilateral inguinal hernia. Ann Surg 2012;255:846-853

19 Koning GG, Wetterslev J, van Laarhoven CJHM, Keus F: The totally extraperitoneal method versus Lichtenstein's technique for inguinal hernia repair: a systematic review with meta-analyses and trial sequential analyses of randomized clinical trials. PLoS One 2013;8:e52599.

20 Bobo Z, Nan W, Qin Q, Tao W, Jianquo L, Xianli H: Meta-analysis of randomized controlled trials comparing Lichtenstein and totally extraperitoneal laparoscopic hernioplasty in treatment of inguinal hernias. J Surg Res 2014;192:409-420.

21 Scheuermann U, Niebisch S, Lyros O, Jansen-Winkeln B, Gockel I: Transabdominal preperitoneal (TAPP) versus Lichtenstein operation for primary inguinal hernia - a systematic review and meta-analysis of randomized controlled trials. BMC Surg 2017;17:55.
22 Köckerling F, Stechemesser B, Hukauf M, Kuthe A, Schug-Pass C: TEP versus Lichenstein: which technique is better for the repair of primary unilateral inguinal hernias in men? Surg Endosc 2016;30:3304-3313.

23 Köckerling F, Bittner R, Kofler M, Mayer F, Adolf D, Kuthe A, Weyhe D: Lichtenstein versus total extraperitoneal patch plasty versus transabdominal patch plasty technique for primary unilateral inguinal hernia repair. Ann Surg 2017;DOI: 10/1097/SLA.0000000000002541.

24 Wake BL, McCormack K, Fraser C, Vale L, Perez J, Grant A: Transabdominal pre-peritoneal (TAPP) vs totally extraperitoneal (TEP) laparoscopic techniques for inguinal hernia repair. Cochrane Database Syst Rev 2005;1:CD004703.

25 Bracale U, Melillo P, Pagnata G, Di Salvo E, Rovani M, Merola G, Pecchia L: Which is the best laparoscopic approach for inguinal hernia repair: TEP or TAPP? A systematic review of the literature with a network meta-analysis. Surg Endosc 2012;26:3355-3366.

26 Antoniou S, Antoniou G, Bartsch D, Fendrich V, Koch OO, Pointner R, Granderath FA: Transabdominal preperitoneal versus totally extraperitoneal repair of inguinal hernia: a meta-analysis of randomized studies. Am J Surg 2013;206:245-252.

27 Wei FX, Zhang YC, Wei H, Zhang YL, Shao Y, Ni R: Transabdominal preperitoneal (TAPP) versus totally extraperitoneal (TEP) for laparoscopic hernia repair: a meta-analysis. Surg Laparosc Endosc Percutan Tech 2015;25:375-383.

28 Köckerling F, Schug-Pass C: Tailored approach in inguinal hernia repair - decision tree based on the guidelines. Front Surg 2014;1:20.

29 Burcharth J, Pommergaard HC, Bisgaard T, Rosenberg J: Patient-related risk factors for recurrence after inguinal hernia repair: a systematic review and meta-analysis of observational studies. Surg Innov 2015;22:303-317.

30 Mikkelsen T, Bay-Nielsen M, Kehlet H: Risk of femoral hernia after inguinal herniorrhaphy. Br J Surg 2002;89: 486-488.

31 Burcharth J, Andresen K, Pommergaard HC, Bisgaard T, Rosenberg J: Direct inguinal hernias and anterior surgical approach are risk factors for female inguinal hernia recurrences. Langenbecks Arch Surg 2014;399:71-76.

32 Andresen K, Bisgaard T, Kehlet H, Wara P, Rosenberg $\mathrm{J}$ : Reoperation rates for laparoscopic vs open repair of femoral hernias in Denmark: a nationwide analysis. JAMA Surg 2014;149:853-857.

33 Henriksen NA, Thorup J, Jorgensen LN: Unsuspected femoral hernia in patients with a preoperative diagnosis of recurrent inguinal hernia. Hernia 2012;16:381-385.

34 Sarli L, Iusco DR, Sansebastiano G, Costi R: Simultaneous repair of bilateral inguinal hernias: a prospective, randomized study of open, tension-free versus laparoscopic approach. Surg Laparosc Endosc Percutan Tech 2001;11:262-267.

35 Mahon D, Decadt B, Rhodes M: Prospective randomized trial of laparoscopic (transabdominal preperitoneal) vs open (mesh) repair for bilateral and recurrent inguinal hernia. Surg Endosc 2003;17:1386-1390.

36 Feliu X, Claveria R, Besora P, Camps J, Fernández-Sallent E, Vinas C, Abad JM: Bilateral inguinal hernia repair: laparoscopic or open approach? Hernia 2011;15:15-18.

37 Gainant A, Geballa R, Bouvier S, Cubertafond P, Mathonnet M: Prosthetic treatment of bilateral inguinal hernias via laparoscopic approach or Stoppa procedure. Ann Chir 2000;125:560-565.

38 Krähenbühl L, Schäfer M, Schilling M, Kuzinkovas V, Büchler MW: Simultaneous repair of bilateral groin hernias: open or laparoscopic approach? Surg Laparosc Endosc 1998;8:313-318.

39 Köckerling F, Schug-Pass C, Adolf D, Keller T, Kuthe A: Bilateral and unilateral total extraperitoneal inguinal hernia repair (TEP) have equivalent early outcomes: analysis of 9,395 cases. World J Surg 2015;39:1887-1894.
40 Jacob DA, Hackl JA, Bittner R, Kraft B, Köckerling F: Perioperative outcome of unilateral versus bilateral inguinal hernia repairs in TAPP technique: analysis of 15,176 cases from the Herniamed Registry. Surg Endosc 2015;29:3733-3740.

41 Nordin P, Haapaniemi S, van der Linden W, Nilsson E: Choice of anesthesia and risk of reoperation for recurrence in groin hernia repair. Ann Surg 2004;240:187192.

42 Westin L, Wollert S, Ljungdahl M, Sandblom G, Gunnarsson U, Dahlstrand U: Less pain 1 year after total extra-peritoneal repair compared with Lichtenstein using local anesthesia: data from a randomized controlled clinical trial. Ann Surg 2016;263:240-243.

43 Dhankhar DS, Sharma N, Mishra T, Kaur N, Singh S, Gupta S: Totally extraperitoneal repair under general anesthesia versus Lichtenstein repair under local anesthesia for unilateral inguinal hernia: a prospective randomized controlled trial. Surg Endosc 2014;28:996-1002.

44 Goel A, Bansal A, Singh A: Comparison of local versus spinal anesthesia in long standing open inguinal hernia repair. Int Surg J 2017;4:3701-3704.

45 Goyal P, Kumar Sharma S, Singh Jswal K, Goyal S, Ahmed M, Sharma G, Pandotra P: Comparison of inguinal hernia repair under local anesthesia versus spinal anesthesia. J Med Dent Sci 2014;13:54-59.

$46 \mathrm{Li}$ J, Ji Z, Li Y: Comparison of laparoscopic versus open procedure in the treatment of recurrent inguinal hernia: a meta-analysis of the results. Am J Surg 2014;207: 602-612.

47 Dedemadi G, Sgourakis G, Radtke A, Dounavis A, Gockel I, Fouzas I, Karaliotas C, Anagnostou E: Laparoscopic versus open mesh repair for recurrent inguinal hernia: a meta-analysis of outcomes. Am J Surg 2010;200:291-297.

48 Karthikesalingam A, Markar SR, Holt PJ, Praseedom RK: Meta-analysis of randomized controlled trials comparing laparoscopic with open mesh repair of recurrent inguinal hernia. Br J Surg 2010;97:4-11.

49 Yang J, Tong da N, Yao J, Chen W: Laparoscopic or Lichtenstein repair for recurrent inguinal hernia: meta-analysis of randomized controlled trials. ANZ J Surg 2013;83:312-318.

50 Pisanu A, Podda M, Saba A, Porceddu G, Uccheddu A: Meta-analysis and review of prospective randomized trials comparing laparoscopic and Lichtenstein technique in recurrent inguinal hernia repair. Hernia 2015; 19:355-366.

51 Köckerling F, Bittner R, Kuthe A, Hukauf M, Mayer F, Fortelny R, Schug-Pass C: TEP or TAPP for recurrent inguinal hernia repair - register-based comparison of the outcome. Surg Endosc 2017;31:3872-3882.

52 Köckerling F, Bittner R, Kuthe A, Stechemesser B, Lorenz R, Koch A, Reinpold W, Niebuhr H, Hukauf M, Schug-Pass C: Laparo-endoscopic versus open recurrent inguinal hernia repair: should we follow the guidelines? Surg Endosc 2017;31:3168-3185.

53 Köckerling F, Jacob D, Wiegank W, Hukauf M, SchugPass C, Kuthe A, Bittner R: Endoscopic repair of primary versus recurrent male unilateral inguinal hernias: are there differences in the outcome? Surg Endosc 2016;30:1146-1155.

54 Köckerling F, Koch A, Lorenz R, Reinpold W, Hukau $M$, Schug-Pass C: Open repair of primary versus recurrent male unilateral inguinal hernias: perioperative complications and 1-year follow-up. World J Surg 2016;40:813-825.

55 Deeba S, Purkayastha P, Paraskevas T, Athanadious A, Darzi A, Zacharakis E: Laparoscopic approach to incarcerated and strangulated inguinal hernias. JSLS 2009;13:327-331.

56 Sartelli M, Coccolini F, van Ramshorst GH, et al: WSES guidelines for emergency repair of complicated abdominal wall hernias. World J Emerg Surg 2013;8:50. 